\title{
Duramycin effects on voltage-gated ion channels: a QT-prolongation risk?
}

Eva-Maria Zebedin, Xaver Koenig, Miroslav Radenkovic, Halyna Pankevych, Hannes Todt, Michael Freissmuth and Karlheinz Hilber*

Address: Center of Biomolecular Medicine and Pharmacology, Institute of Pharmacology, Medical University of Vienna, Austria

Email: Karlheinz Hilber* - karlheinz.hilber@meduniwien.ac.at

* Corresponding author

\author{
from I3th Scientific Symposium of the Austrian Pharmacological Society (APHAR). Joint Meeting with the Austrian Society of Toxicology (ASTOX) and the \\ Hungarian Society for Experimental and Clinical Pharmacology (MFT) \\ Vienna, Austria. 22-24 November 2007 \\ Published: 14 November 2007 \\ BMC Pharmacology 2007, 7(Suppl 2):A9 doi:10.1 186/147/-2210-7-S2-A9
}

This abstract is available from: http://www.biomedcentral.com/I47I-22/0/7/S2/A9

(c) 2007 Zebedin et al; licensee BioMed Central Ltd.

Drug-induced prolongation of the QT interval has become a major safety concern during drug development. Duramycin, a peptide antibiotic, is in clinical development for the treatment of cystic fibrosis. It becomes deposited in cellular membranes where it binds to phosphatidylethanolamine. Duramycin may thereby change biophysical membrane properties and perturb ion channel function. Thus, its application possibly carries the risk to elicit a QT-prolongation. Here, we tested the effects of duramycin on currents through voltage-gated hERG potassium, sodium and calcium channels of various mammalian cell types in whole cell patch clamp studies. We found that duramycin bath concentrations between 1 $\mathrm{nM}$ and $0.1 \mu \mathrm{M}$ did not generate any effects on these currents. Concentrations $\geq 0.3 \mu \mathrm{M}$, however, reduced the amplitudes of all the investigated currents. Moreover, sodium current fast inactivation kinetics was slowed in the presence of duramycin. The described effects exhibited concentration-dependency. A further rise in duramycin bath concentration $(\geq 3.3 \mu \mathrm{M})$ induced a leak current consistent with pore formation. The reported effects of duramycin on ion channel function may be generated by disruption of biophysical membrane properties rather than by specific interaction with ion channel proteins. Our data suggest that, under therapeutic conditions (i.e. administration via inhalation), duramycin is unlikely to elicit a QT-prolongation. 